\title{
How Mobility Models Affect The Design of Network Coding Schemes for Disruption Tolerant Networks
}

\author{
M. Chuah, P. Yang, Y. Xi \\ Department of Computer Science \& Engineering \\ Lehigh University \\ chuah@cse.lehigh.edu, pey204@1ehigh.edu, yox205@1ehigh.edu
}

\begin{abstract}
Mobile nodes in some challenging network scenarios suffer from intermittent connectivity and frequent partitions e.g. battlefield and disaster recovery scenarios. Disruption Tolerant Network (DTN) technologies are designed to enable nodes in such environments to communicate with one another. Recently, network coding schemes for DTN has been proposed. However, these schemes work well only in scenarios with homogeneous mobility models. In this paper, we propose an efficient context-aware network coding (CANCO) scheme for DTNs that achieves higher delivery ratio than the existing published network coding schemes in networks where nodes move according to non-homogeneous mobility models. We also show that our scheme delivers $300 \%$ more messages with higher data efficiency than a MORE-like scheme because our scheme does not suffer from the stop-andwait feature in the MORE protocol.
\end{abstract}

\section{INTRODUCTION}

With the advancement in technology, many users carry computing devices e.g. PDAs, cell-phones etc with wireless interfaces. Such devices can form mobile ad hoc networks and communicate with one another via the help of intermediate nodes. Such ad hoc networks are very useful in several scenarios e.g. battlefield operations, vehicular ad hoc networks and disaster response scenarios. Many ad hoc routing schemes have been designed for ad hoc networks but such routing schemes are not useful in some challenging network scenarios where the nodes have intermittent connectivity and suffer frequent partitioning. Recently, disruption tolerant network technologies [1],[2] have been proposed to allow nodes in such extreme networking environment to communicate with one another. Several DTN routing schemes [3],[4] have been proposed. In addition, some researchers have proposed using randomized network coding [9],[10] technique to enhance the delivery performance of a batch of messages in DTNs. These existing schemes are designed assuming the nodes move according to a homogeneous mobility model and hence may not work well if the nodes move according to a non-homogeneous mobility model. Thus, in this paper, we explore some network-coding schemes that will work better when the nodes move according to a non-homogeneous mobility model. Our schemes allow coded packets to be distributed to more popular nodes and hence can achieve higher message delivery ratio than those schemes that are designed assuming a homogeneous mobility model. We conduct extensive simulation studies for unicast communications to demonstrate the superiority of our designed schemes. In the CANCO scheme we design, an intermediate node uses a friendliness metric to decide if it wishes to spread coded packet to another node it encounters. Thus, the friendliness threshold can be set such that only those popular nodes that can meet more nodes receive coded packets and hence increases the chance of a receiver receiving enough coded packets to reconstruct a message before the message expires. In addition, unlike the MORE scheme [7], our CANCO scheme allows a source node to keep disseminating coded packets for new messages as soon as they are generated without having to wait for an acknowledgement packet back from a receiver. Thus, it does not suffer any throughput degradation due to the stop-and-wait approach used in the MORE scheme [7]. In summary, our contributions in this paper include (a) design of a network coding scheme that performs better in sparse networks where nodes move according to nonhomogeneous mobility models, (b) evaluating network coding schemes using more realistic mobility models e.g. community-based models, dieselnet models constructed using real mobility traces, (c) comparison of our scheme with other schemes.

The rest of the paper is organized as follows: In Section II, we discuss several areas of related work, namely some existing DTN unicast and multicast routing schemes, and network coding schemes designed for DTNs. In Section III, we describe the system model we assume in this work. In Section IV, we describe how our context-aware network coding (CANCO) scheme and two variants of this scheme work. In Section V, we first describe the simulation model we use and then present extensive simulation studies we conduct to demonstrate the superiority of the CANCO scheme.

\section{RELATED WORK}

Previous studies have proposed to use erasure coding to deal with network disruptions in DTNs [5],[6]. It has been shown that network coding [7] can improve the throughput in wireless communication. However, in DTNs, a node seldom has more than one neighbor, and such wireless coding opportunities rarely occur. In [9], the authors propose a scheme called the network coding based epidemic routing (NCER) scheme which transmits a batch of data packets with network coding. In this scheme, when two nodes meet, they transmit coded packets to each other. A coded packet $\mathrm{x}$ is a linear combination of $\mathrm{K}$ source 
packets, $\mathrm{E}_{1} \ldots \mathrm{E}_{\mathrm{K}}$ in the form $x=\sum_{i=1}^{K} \alpha_{i} E_{i}$ where $\alpha_{\mathrm{i}}$ is the coding coefficient. Suppose that node a holds $\mathrm{m}$ coded packets in its buffer, node a encodes all coded packets in its buffer, namely $\mathrm{x}_{1} . . \mathrm{x}_{\mathrm{m}}$ to generate a coded packet $\mathrm{x}_{\mathrm{a}}$ :

$$
x_{a}=\sum_{i=1}^{m} \beta_{i} x_{i}
$$

where all multiplication and addition operations are defined on a Galois Field and is randomly chosen from the field. Node $a$ then transmits $\mathrm{x}_{\mathrm{a}}$ along with its coding coefficients over the original packets to node $b$. When node $b$ receives $x_{a}$, it stores $x_{a}$ in its buffer if space is available. Otherwise, node $b$ encodes $\mathrm{x}_{\mathrm{a}}$ with each packet in its buffer as follows: $x_{i}^{\prime}=x_{i}^{\prime}+\gamma_{i} x_{a}$, where $x_{i}^{\prime}$ represents the $\mathrm{i}^{\text {th }}$ coded packet in the buffer of node $b$, and $\gamma_{i}$ is randomly chosen from the Galois Field.

The destination obtains a coded packet when it meets another node, and attempts the decoding process to retrieve $\mathrm{K}$ source packets after $\mathrm{K}$ coded packets have been collected. Because the coding coefficients and the coded packets are known, each coded packet represents a linear equation with the $\mathrm{K}$ source packets as unknown variables. Decoding the $\mathrm{K}$ source packets is equivalent to solving the linear system composed of $\mathrm{K}$ coded packets. In NCER, the nodes keep exchanging the packets until they receive an ACK from the destination that all $\mathrm{K}$ packets have been received or the TTLs of the packets have expired. Thus, this scheme is not quite efficient.

In [10], the authors propose another scheme called the efficient network coding protocol (E-NCP). In this scheme, the source transmits slightly more than $\mathrm{K}$ coded packets such that these coded packets are sufficient to decode the original packets with high probability. All these coded packets are referred to as pseudo source packets. Each pseudo source packet is then disseminated to $\mathrm{L}$ random nodes in the network in the same spirit as the binary spraying scheme in [10]. The authors in [10] have shown that binary spraying is the optimal spraying method with the minimal packet transmission delay under a homogenous mobility model. By adjusting L, one can tune the trade off between the number of relay transmissions and packet transmission delay.

\section{SYSTEM MODEL}

In this work, we consider a disruption tolerant network consisting of $\mathrm{N}$ mobile nodes. The nodes may move according to a homogeneous or non-homogeneous mobility model. The homogeneous models consider in this work include the RWP model and some realistic mobility models e.g. the Zebranet [5] and UMassDieselNet models [4]. The non-homogeneous model considered is the Communitybased model [8].

For unicast communications, we assume that messages are sent from a source to a destination. Each source periodically sends messages, each of which consist of $\mathrm{K}$ packets destined to a destination. Each message has an expiration time (denote as Texp) and all coded packets within the same message have the same Texp. A transmission opportunity arises when a pair of nodes "meet" i.e. they are within the transmission range of each other. Each node has a buffer size of B packets. The packets in the buffer can be purged at any time upon the expiration of the packets.

\section{NeTwork Coding Schemes}

In this section, we first describe the erasure coding (EC) scheme introduced in [5]. Then, we describe two network coding schemes that we design to transmit a batch of data packets from a source to a destination in DTNs. In the EC scheme, a source codes a message, divides it into kr chunks, distributes them to $\mathrm{kr}$ relays. A destination only needs to receive $\mathrm{k}$ chunks to reconstruct the message. We use $\mathrm{r}=2$ and $\mathrm{k}=16$ in this work.

The first network coding scheme we design is called the network coding with binary spread (NCBS) scheme. In this scheme, an intermediate node with $\mathrm{m}$ coded packets of a message will spread half of its packets to another node it encounters. Such a process continues until an intermediate node only has one coded packet of that message or it meets the destination. If it meets the destination, it sends all its coded packets to the destination. If an intermediate node only has one coded packet of that message, then it will hold on to that packet until it meets the destination. One may consider a variant of the NCBS scheme which allows the source or any intermediate node to spray its coded packets only if its batch size exceeds a certain minimum value. This variant is referred to as the NCBS with a Minimum Batchsize (NCBS-MB) scheme. If the minimum batch size (MBS) is set at 0 , then the NCBS-MB scheme is the same as the NCBS scheme.

The NCBS scheme works well in a homogeneous mobility model but does not work well in a nonhomogeneous model. For example, some coded packets may be passed to a locally moving node which has a very small chance of meeting the destination. Thus, we design a scheme where an intermediate node uses the friendliness metric, and the delivery predictability value to decide if it wants to spread half of its coded packets to a node it encounters, or do nothing. We refer to this scheme as the context-aware network coding (CANCO) scheme. The friendliness metric measures how popular a node is while the delivery predictability estimates the probability of reaching another node. The friendliness metric helps to distinguish between globally and locally moving nodes. The delivery predictability metric is used to distinguish nodes that are closer to the destination. In CANCO, the delivery predictability metric of a node to the destination is computed in the same manner as in the Prophet [3] routing scheme. Each node periodically sends a beacon that contains its delivery predictabilities to all other nodes in the network. Each node that hears another node's beacon updates its own delivery predictabilities according to the 
following three equations:

$$
\begin{array}{cc}
P(a, b)=P(a, b)_{\text {old }}+\left(1-P(a, b)_{\text {old }}\right)^{*} \alpha & \mathrm{Eq}(1 \mathrm{a}) \\
P(a, b)=P(a, b)_{\text {old }} \times \gamma^{k} & \mathrm{Eq}(1 \mathrm{~b}) \\
P(a, c)=P(a, c)_{\text {old }}+\left(1-P(a, c)_{\text {old }}\right) * P(a, b)^{*} P(b, c) * \beta & \mathrm{Eq}(1 \mathrm{c}) \\
\text { In [3], } \alpha \text { is set to } 0.75, \beta \text { is set to } 0.25 \text { and } \gamma \text { is set to } 0.98 .
\end{array}
$$

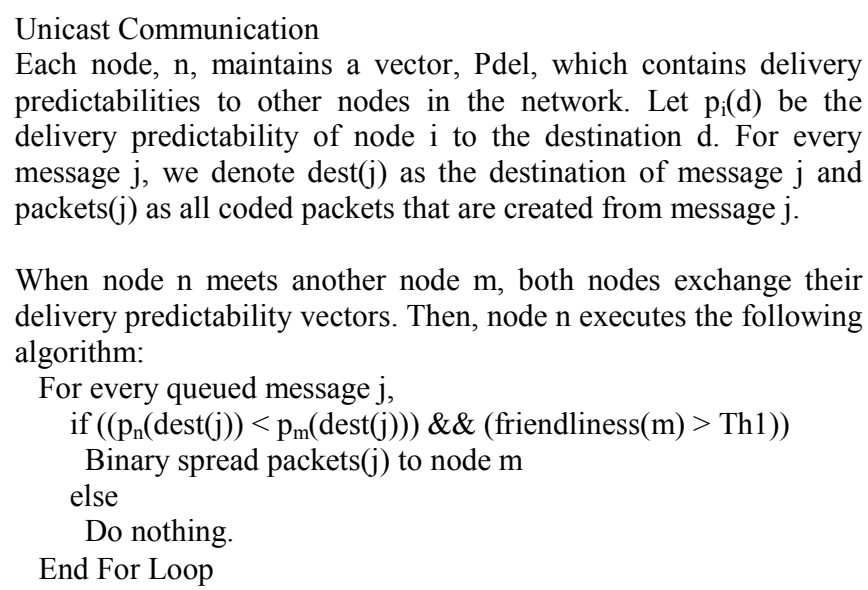

When node $\mathrm{n}$ meets another node $\mathrm{m}$, both nodes exchange their delivery predictability vectors. Then, node $\mathrm{n}$ executes the following algorithm:

For every queued message $\mathrm{j}$, if $\left(\left(p_{n}(\operatorname{dest}(j))<p_{m}(\operatorname{dest}(j))\right) \& \&(\right.$ friendliness $\left.(m)>T h 1)\right)$ Binary spread packets $(\mathrm{j})$ to node $\mathrm{m}$

Figure 1: Pseudo Code for Unicast Communication using the CANCO scheme.

The pseudo code for the CANCO scheme is shown in Figure 1. The main difference between the CANCO and the NCBS scheme is that the NCBS scheme does not use the friendliness metric. Similar to the NCBS scheme, we can also set a minimum batch size beyond which the source or intermediate node will not spray the coded packets they have to other nodes. We refer to this as the CANCO-MB scheme.

Our scheme is expected to perform better than the MORE scheme since the source is allowed to send coded packets from a new message once it is done with transmitting the coded packets of one message. The MORE protocol behaves more like a stop-and-wait protocol where a source will keep sending coded packets for a particular message until it receives an acknowledgement from its receiver. We can also add a sliding window with a window size Nmax so that only Nmax outstanding unacknowledged messages are allowed. We refer to this variant as the CANCO-SW scheme. We will compare the CANCO scheme, the CANCO-SW scheme, and the MORE-like scheme in the next section.

\section{PERFORMANCE EVALUATION}

\section{A. Simulation Setup}

To investigate the usefulness of the three network coding schemes we describe in Section IV, we implement the EC, the NCBS, the and CANCO schemes in NS-2 version 2.27 [12]. We assume that the wireless bandwidth is $2 \mathrm{Mb} / \mathrm{s}$ and the transmission range is $250 \mathrm{~m}$.

Network Model: In our default network scenario, we assume that there are 40 nodes randomly distributed within a geographical size of $3000 \times 3000 \mathrm{~m}^{2}$. We also have results for a larger network with 100 nodes. Interested readers can refer to [12] for these results.

Node Movement Model: The nodes move either according to (a) the random waypoint (RWP) mobility model, (b) the Zebranet [5] model, (c) the UMassDieselNet model [4], or (d) the Community-based model [8]. For the RWP model, each node selects a random destination, and moves towards the destination with a speed chosen randomly between (vmin, vmax) $\mathrm{m} / \mathrm{s}$. After the node reaches its destination, it pauses for a period of time and repeats this movement pattern. Unless otherwise stated, vmin is set to $1 \mathrm{~m} / \mathrm{s}$, and vmax is set to $5 \mathrm{~m} / \mathrm{s}$ for all nodes in the homogeneous model. For the Zebranet model [5], we create a semisynthentic model as follows: we synthesize node speed and turn angle distributions from the observed data and create other node-movements using the same distribution. We use both distance and time scaling to fit the original data found in the trace into the network environment that we are interested in. The average node speed in the Zebranet model is $6 \mathrm{~m} / \mathrm{s}$. The UmassDieselNet model is constructed from the traces collected in UMass DieselNet project. For the Community-based (CB) model, $50 \%$ of the nodes move locally (within $1 \%$ of the whole area) and $50 \%$ of the nodes move globally (i.e. each moves within the whole area). From the $\mathrm{CB}$ traces we generated, we observe that the average number of nodes seen by a local node within $1000 \mathrm{~ms}$ is 4.3 while the average number of nodes seen by a global node within $1000 \mathrm{~ms}$ is 7.5 . Thus, we set the threshold Th1 to 6 .

Data Item Generation Model: For unicast scenarios, we assume that there are four sessions, each generating 1 message every 200s. By default, each message has 1000 packets. We refer to these as the source packets. For the network coding related schemes, a total of 2000 coded packets are generated from these 1000 source packets. A destination only needs to get 1000 of the 2000 coded packets to decode the 1000 source packets. For the erasure coding scheme, we assume that a message is encoded and divided into 32 chunks and only 16 chunks are required to reconstruct a message.

The performance metrics that we use to compare different combinations of schemes are

- Delivery Ratio, Psucc-this is the number of messages successfully received by a destination.

- Average message delivery latency - this is the average time it takes for the destination (or a receiver) to receive a message successful.

- Data efficiency - this is measured as the number of useful data bytes over the number of total transmitted data bytes (does not include control overhead)

Each data point reported in the simulation results section is the average of 5-10 runs. 


\section{B. Simulation Results for Unicast Communication}

\section{1) Impact of Message Expiration Time}

In our first experiment, we compare the performance of the CANCO, the NCBS, and the Erasure Coding (EC) schemes. The nodes either move according to the UMassDieselNet or the CB model. In Figures 3(a), 3(b), $3(\mathrm{c})$, we plot the delivery ratio, the average message delivery latency, and the data efficiency when the $\mathrm{CB}$ model is used.

We see from Figures 3(a),3(b), and 3(c) that the CANCO scheme performs the best. The interesting observation is that NCBS performs better than the EC scheme until Texp is about $5.5 \mathrm{hr}$. Then, the EC scheme performs better than the NCBS scheme. This may be due to the fact that the coded packets are spread to too many nodes in the NCBS scheme, and hence it takes longer for a destination to collect at least 1000 coded packets. In the $\mathrm{EC}(2,16)$ scheme, there are at most 32 relaying nodes, and a destination only needs to encounter 16 of them to receive enough data chunks to reconstruct a message.

The average message delivery time for the EC scheme is horribly long because the source takes at least 16 encounters to spread 16 chunks, and the destination needs to get 16 chunks from these intermediate nodes, or meets the source node (which has very small probability in the CB-model). For those messages that are delivered in both the NCBS and CANCO schemes, the average delivery latency is smaller in the CANCO scheme. This is because CANCO scheme uses fewer hops to deliver the coded packets and by using better paths, intermediate nodes with coded packets can reach destination faster and hence the average batch size received per encounter at the destination is larger. In the NCBS scheme, a node binary spreads the coded packets to any node it encounters without considering the movement characteristics of that node. When the coded packets are spread to locally moving nodes, they have slim chances of reaching the destination node and hence the delivery ratio remains low in the NCBS scheme. However, in the CANCO scheme, both the friendliness metric and the delivery predictability values are used to decide whether an intermediate node will binary spread its coded packets to a node it encounters. Thus, we see that for the CANCO scheme, the delivery ratio achieved in the $\mathrm{CB}$ model is much better than the NCBS scheme There are some messages that are delivered in the CANCO scheme but not in the NCBS scheme. These messages usually have higher delivery latency (that's why they are not successful in the NCBS scheme because they expire). Such messages sometimes cause the overall message delivery latency for the CANCO scheme to be slightly higher for smaller Texp values.

The data efficiency results indicate that CANCO has good data efficiency. The high efficiency value shown for the EC scheme is misleading since we do not take into considerations the unsuccessful transmissions. Those messages that are delivered in the CANCO and NCBS schemes but not the EC scheme usually take more hops. In addition, we did not implement a feature where nodes maintain a list of the sequence numbers of delivered messages so that they can inform other nodes they encounter to remove these messages from the buffer. Thus, the data efficiency for the NCBS and CANCO scheme becomes poorer than the EC scheme when Texp exceeds $4.5 \mathrm{hr}$.

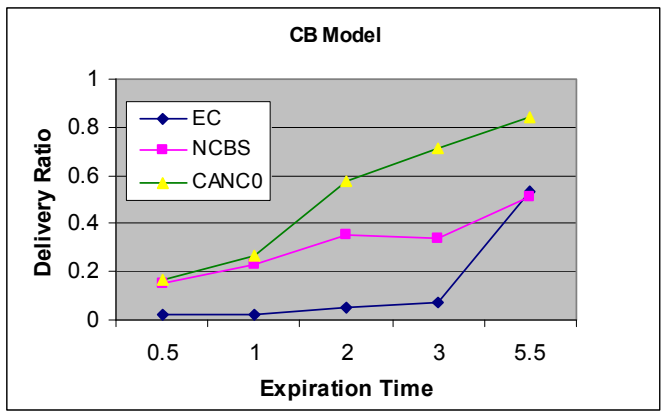

Figure 3(a) Delivery Ratio vs Texp (CB model)

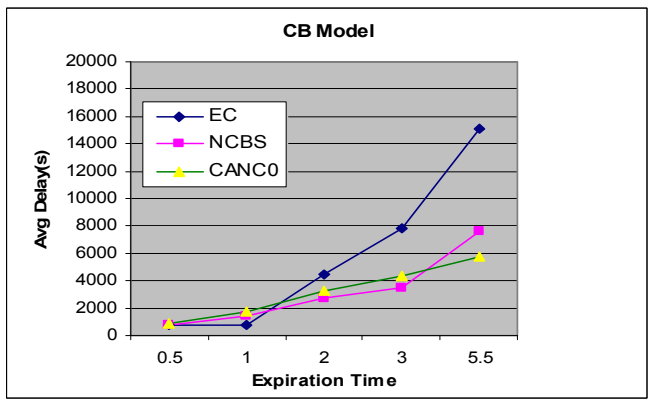

Figure 3(b): Avg Message Delivery latency vs Texp

Since some messages fail because the coded packets are spread to too many nodes, we anticipate that the delivery performance may be better if we set a minimum batch size (MBS) beyond which a node will not binary spread its coded packets. Thus, we repeat the experiment using the $\mathrm{CB}$ model and the CANCO scheme but set the minimum batch size to 100 .

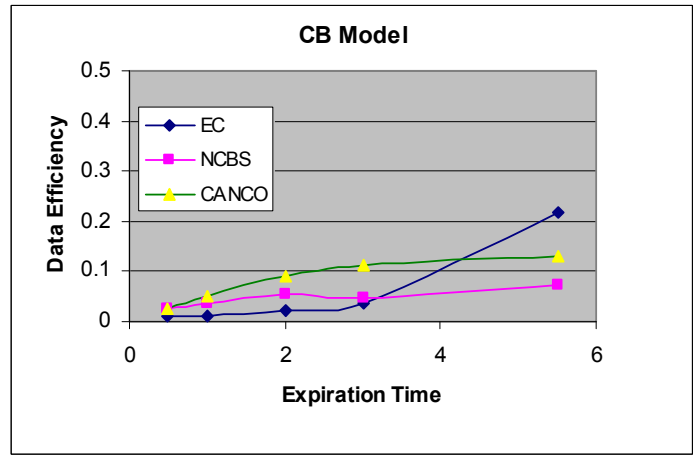

Figure 3(c) Data Efficiency vs Texp(CB-Model)

We have results that compare the CANCO scheme $(\mathrm{MBS}=1)$ with the CANCO-MB scheme $(\mathrm{MBS}=100)$. Due to space limitation, we did not include them here. Our results indicate that the CANCO-MB scheme achieves 5$10 \%$ higher delivery ratio than the CANCO scheme. The CANCO-MB scheme performs better because it sets a minimum batch size beyond which the global nodes are not allowed to spray the coded packets further. Thus, a receiver can receive larger batches of packets from globally moving 
nodes and can receive 1000 coded packets faster. The average message delivery latency for those messages that are delivered is smaller with a minimum batch size of 100 . However, since those messages that are not delivered in the CANCO scheme but in the CANCO-MB scheme take longer time, the overall average delay for the CANCO-MB scheme may be slightly higher. The data efficiency for the CANCO-MB scheme is $25 \%$ better than that for the CANCO scheme.

\section{2) Impact of Mobility Model}

In this section, we are interested in exploring how the delivery performance of the CANCO scheme differs with different mobility models. The different mobility models we use in this section are: the RWP, ZebraNet, UMassDieselNet and CB models. The RWP, ZebraNet, UMassDieselNet mobility models are homogeneous mobility models while the CB model is a non-homogeneous model. Recall that in the CANCO scheme, we use a friendliness metric to decide if packets should be sprayed to a newly encountered node. For the homogeneous models e.g. the RWP, ZebraNet and UMassDieselNet models, we set Th1 to be 0 . However, we set Th1 to be 6 for the CB model. The minimum batch size is set to 1 . The results for the delivery ratio, the average message latency, and the data efficiency are plotted in Figures 4(a), 4(b), and 4(c) respectively.

The results show that the delivery performance of the CANCO scheme degrades when the UMassDieselNet or the $\mathrm{CB}$ model is used. The delivery ratio for the RWP and Zebranet models is $80 \%$ and $100 \%$ respectively when Texp is $1 \mathrm{hr}$. However, it is only $27 \%$ and $39 \%$ for the CB and UMassDieselNet (UMDN) model respectively. The contact duration (CDur), the node encounter time (NET), and the intercontact time (ICT) for the RWP, Zebranet, UMassDieselNet and the CB (between locally and gobally moving nodes) models are shown in Table 1 . The node encounter time is the time in between a node making contacts with any node while the inter-contact time is the time interval in between two nodes making contacts with each other. As we can see, the node encounter and the intercontact times for the UMDN or the $\mathrm{CB}$ model are much longer than what are observed in the ZebraNet or RWP model. For example, the NETs for the CB and UMDN models are 2.5 to 3 times longer than that for the RWP model. Thus, the observed delivery performance for these two models is poorer.

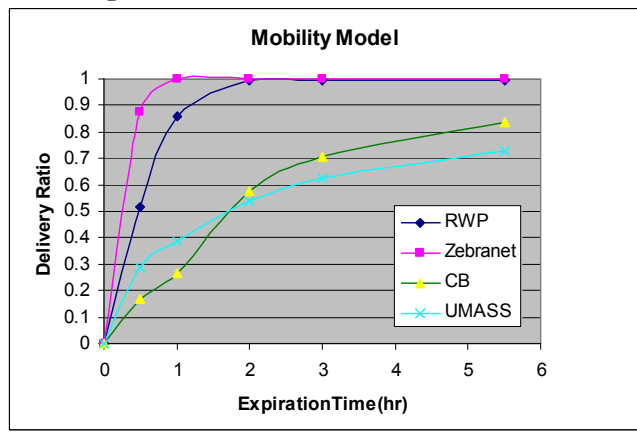

Figure 4(a) Delivery Ratio vs Texp

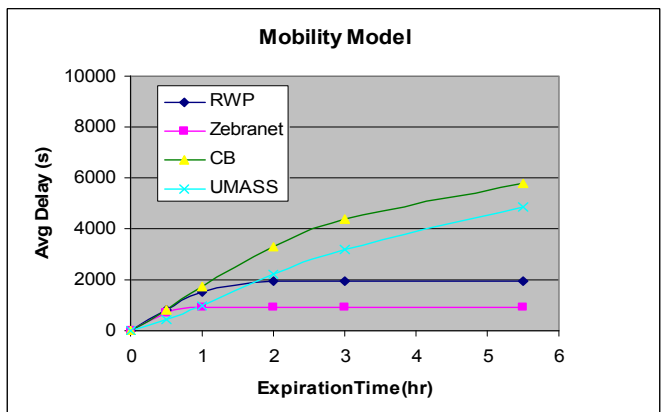

Figure 4(b) Avg Message Latency vs Texp

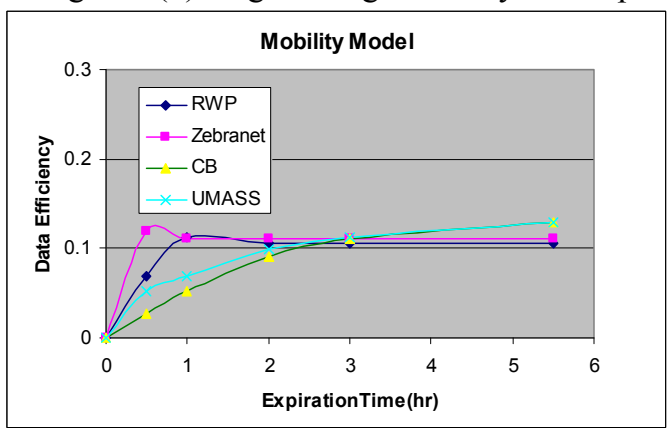

Figure 4(c): Data Efficiency vs Texp

\begin{tabular}{|l|l|l|l|l|}
\hline $\begin{array}{l}\text { Mean } \\
\text { Value }\end{array}$ & RWP & Zebranet & UMDN & CB \\
\hline CDur (s) & 113.4 & 26.0 & 771.4 & 115.4 \\
\hline ICT(s) & 2496.2 & 897.2 & 3931.1 & 3626.2 \\
\hline NET (s) & 103.6 & 50.1 & 312.6 & 252.9 \\
\hline
\end{tabular}

Table 1: Contact Characteristics of different models.

\section{Comparison of Various Schemes}

In this section, we compare the CANCO scheme, the CANCO with Sliding Window (CANCO-SW) scheme and the MORE-like (MORE-SW) scheme. The main difference between the CANCO-SW and the MORE-SW scheme is that in the MORE-SW scheme, a intermediate node spreads the coded packet to any node it encounters while in CANCO-SW scheme, it only spreads to a node with a friendliness metric that exceeds a certain threshold. Using the CB model, the threshold is chosen such that only global nodes are involved in spreading coded packets from the source to the destination. We use the network scenario with 100 nodes distributed over $4750 \times 4750 \mathrm{~m}^{2}$ and where the nodes move according to the $\mathrm{CB}$ model. Four unicast flows are used. Each flow generates messages at rate of 1 $\mathrm{msg} / 200 \mathrm{~s}$. For the schemes using the sliding window, we set the window size, Nmax, to be 100 , or 50 .

The results we obtained for the average delivery ratio, the average message latency and the average data efficiency are plotted in Figures 5(a), 5(b), and 5(c) respectively. The results indicate that the CANCO scheme achieves similar performance as the CANCO-SW $\left(\mathrm{Nmax}_{100}=100\right.$ but outperforms CANCO-SW $(\mathrm{Nmax}=50)$ by achieving higher delivery ratio, lower latency and higher data efficiency. This shows that the source can take more advantage of opportunistic transmissions when no limit is set to the maximum number of outstanding unacknowledged messages. The CANCO-SW scheme performs better than 
the MORE-SW scheme because friendliness metric is used to choose more popular nodes as the relaying nodes.

\section{CONCLUDING REMARKS}

In this paper, we have presented two network coding schemes, namely the (a) NCBS, (b) CANCO schemes, and compare their performances using different mobility models and message expiration time. Our results indicate that the CANCO scheme performs better than the NCBS and EC schemes especially when nodes move according to nonhomogeneous models e.g. the $\mathrm{CB}$ model. Our results also indicate that the delivery performance can be improved by limiting the minimum batch size beyond which the intermediate node does not spray coded packets. In addition, we have compared the CANCO scheme with a MORE-like scheme and show that the delivery performance using the CANCO scheme can be as much as $300 \%$ better than that can be achieved for a MORE-like scheme with Nmax $=50$ which does not use the friendliness metric. In this work, we only consider unicast communications. We intend to investigate how our context-aware network coding scheme perform using more realistic human-based mobility traces [13], and for multicast scenarios. In addition, we also intend to implement and evaluate our scheme in a small testbed.

\section{ACKNOWLEDGMENT}

This work has been supported by DARPA under Contract W15P7T-06-C-P430. Any opinions, findings, and conclusions or recommendations expressed in this paper are those of the authors and do not necessarily reflect the views of the sponsor of this work.

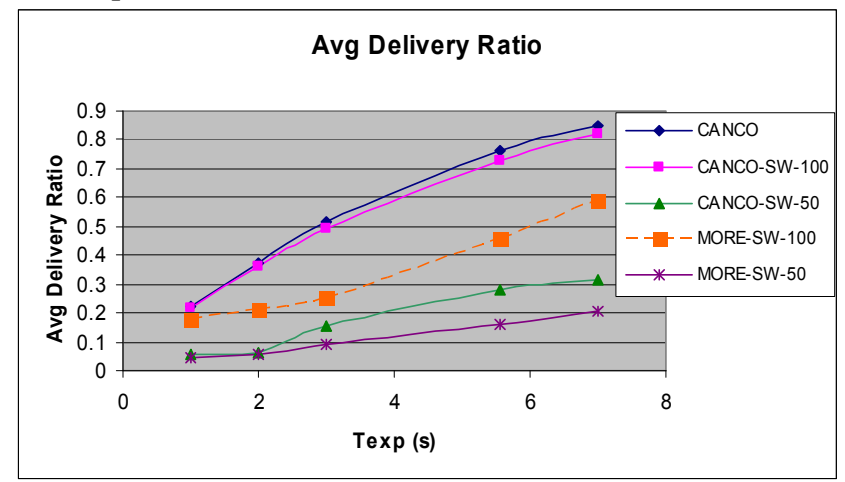

Figure 5(a): Average Delivery Ratio vs Texp (s)

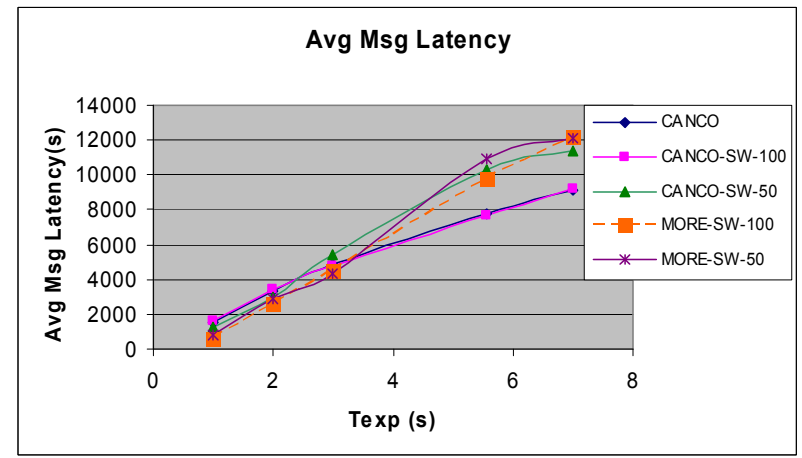

Figure 5(b): Average Message Latency vs Texp(s)

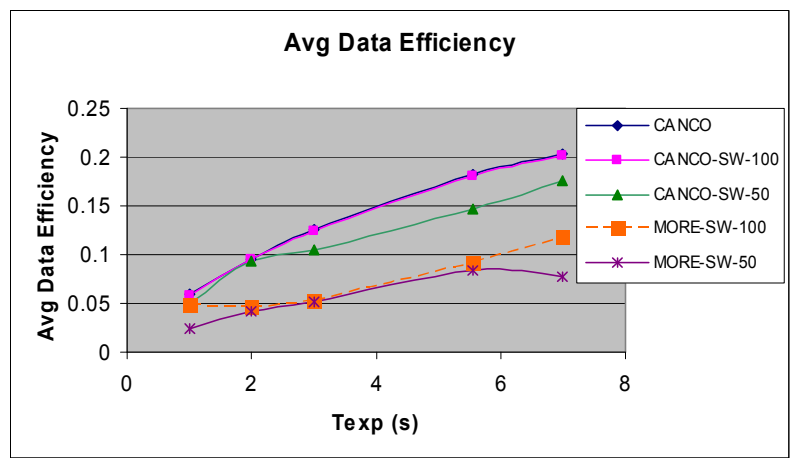

Figure 5(c): Average Data Efficiency vs Texp

\section{REFERENCES}

[1] K. Fall, “ A delay tolerant network architecture for challenged networks", Proceedings of ACM Sigcomm, 2003.

[2] V. Cerf et al, "Delay Tolerant Networking Architecture", RFC4838, April, 2007

[3] A. Lingren et al, "Probabilistic Routing in Intermittently Connected Networks", Proceedings of Workshop on Service Assurance with Partial and Intermittent Resources, Aug, 2004.

[4] J. Burgess et al, "MaxProp: Routing for vehicle-based disruption tolerant networks", Proceedings of IEEE Infocom, 2006.

[5] Y. Wang et al, "Erasure-Coding Based Routing for Opportunistic Networks", Proceedings of ACM workshop on WDTN, 2005.

[6] S. Jain, M. Demmer, R. Patra, K. Fall, "Using Redundancy to Cope with Failures in a Delay Tolerant Network", Proceedings of ACM Sigcomm, Philadelphia,PA, Aug, 2005.

[7] S. Katti et al, "XORs in the Air: Practical Wireless Network Coding", Proceedings of ACM Sigcomm, 2006.

[8] T. Spyropoulos et al, "Efficient routing in intermittently connected mobile networks: multiple copy case" to appear in IEEE/ACM Transactions on Networking, 2007.

[9] Y. Lin, B. Liang, B. Li, "Performance Modeling of Network Coding in Epidemic Routing", Proceedings of ACM MobiOpp, 2007.

[10] Y. Lin, B. Li, B. Liang, "Efficient Network Coded Data Transmissions In Disruption Tolerant Networks", Proceedings of IEEE Infocom, April, 2008.

[11] "The network simulator ns-2", [Online] at http://www.isi.edu/nsnam/ns/.

[12] M. Chuah, P. Yang, Y. Xi, "Impact of Mobility Models on the Performance of Network Coding Schemes in DTNs", CSE Technical Report, Jan, 2009.

[13] A. Chaintreau et al, "Impact of Human Mobility on Opportunistic Forwarding Algorithms", IEEE Transaction on Mobile Computing, Vol 6, No 6, pp 606-620, June, 2007. 